\title{
Human Ability Improvement with Wireless Sensors in Human Computer Interaction
}

\author{
Alao, O.D \\ Department of Computer \\ Science \\ School of Computing and \\ Engineering Sciences, \\ Babcock University \\ Ogun State. Nigeria.
}

\author{
Joshua J.V \\ Department of Computer \\ Science \\ School of Computing and \\ Engineering Sciences, \\ Babcock University \\ Ogun State. Nigeria.
}

\author{
Somefun A.A \\ Department of Computer \\ Science \\ School of Computing and \\ Engineering Sciences, \\ Babcock University \\ Ogun State. Nigeria.
}

\begin{abstract}
Wireless sensors are nowadays engaged in almost every human endeavour and has made live more convenient and conducive. Human Computer interaction (HCI), which is about communication between human user and computer system or devices is a field of computer science that is gaining a lot of recognition in the improvement of human abilities. The communication between a person or user and the computer may involve certain device, medium or channel that has to be configured or programmed in a way that will facilitate an efficient interaction.

Wireless sensors in HCI enhances and improves the way of life and the interaction in a wide variety of domains Therefore, it is important to know the specifications of these sensors and how they are being used in order to create human computer interfaces, which tackle complex tasks.

This paper comprehensively carried out an overview of wireless sensors and HCI, security requirements and challenges of wireless sensors and how wireless sensors interrelate with HCI to improve human abilities in their daily activities. Finally, a brief discussion on the future of sensors in HCI is presented.
\end{abstract}

Keywords; Sensors, Human Ability, Human-Computer Interaction, Modality and Activity Sensing

\section{INTRODUCTION}

With sensors employed in almost every aspect of lives, it has improved the well-being of people. Sensor is a device that detects, senses physical stimulus such as heat, light, sound, motion, etc and then respond or react to it in a particular way and transmits a resulting impulse. The application of wireless sensors has greatly improved and imparted utilization of many system units for example, television with remote control or automatic door system. Also, the integration of sensors and networking technologies has enabled the development of new applications in a wide variety of domains such as, smart homes, e-health, and intelligent transport systems. (Paravati and Gatteschi, 2015)

Automatic recognition of physical activities commonly referred to as human activity recognition (HAR) has emerged as a key research area in human-computer interaction (HCI) and mobile and ubiquitous computing. One goal of activity recognition is to provide information on a user's behaviour that allows computing systems to proactively assist users with their tasks. Human activity recognition requires running classification algorithms, originating from statistical machine learning techniques. Mostly, supervised or semi-supervised learning techniques are utilized and such techniques rely on labelled data, i.e., associated with a specific class or activity. In most of the cases, the user is required to label the activities and this, in turn, increases the burden on the user. (Sunny, George and Kizhakkethottam, 2015)

The presence of sensors in wireless technology with components like microprocessor, microcontroller makes technology omnipresent and increasingly embedded into the environment. This brings intelligence into various applications like intelligent factories, smart homes, intelligent building, smart environment etc. Wireless sensor has broken the bond where user can only interact with one stationary device and make rooms for concept where user interacts with the environment. This is made possible through interface technologies like speech interaction, gesture recognition, eyetracking and wearable devices.

The future of human computer interaction systems lies in how intelligently these systems with the use of wireless sensors can take into account the user's context. Furthermore, research on recognizing the daily activities of people has progressed steadily, but little focus has been devoted to recognizing joint activities as well as movements in a specific activity. For many applications such as rehabilitation, sports medicine, geriatric care, and health/fitness, monitoring the importance of combined recognition of activity and movements can drive health care outcome (Varkey, Pompili, \& Walls, 2012)

This paper discusses the role of wireless sensors in facilitating seamless communications between human and computer, a comprehensive review of some application areas of the 
wireless sensors technology in HCI, associated challenge of wireless sensorss and how they interrelate with $\mathrm{HCI}$ to improve human abilities in their daily activities. The paper concludes with a brief discussion on the future of sensors in HCI.

\section{Overview of Sensors}

A sensor is an electronic device used to detect or measure a physical quantity and convert it into an electronic signal. In other words, sensors are devices that translate aspects of physical reality into a representations understandable and can be processed by computers. Fritzsche (2015) defined a sensor is an object performing a sensing task, gathering information about an object or a process, including the occurrence of events. A sensor technically acts as a transducer which transforms physical signals to electrical energy. Sensors link the physical with the digital world by capturing and revealing real-world phenomena and converting these into a form that can be processed, stored, and acted upon (Dargie \& Poellabauer, 2010). Sensors have been known to drive computer automation and vision over the years (Hua, et al., 2017). Sensors are the pedestal components on which object tracking is based; and object tracking, in turn, has been extensively studied in computer vision due to its applications in surveillance, human-computer interaction, video indexing, and traffic monitoring, to name a few.

Sensors continue to rapidly evolve, becoming increasingly smaller, cheaper, more accurate, more reliable, efficient, responsive and with increasing communication capability. These key factors as well as the availability of new technologies are contributing to the growth of the market of consumer electronics sensors, thus reducing their costs. This scenario has fostered the embedding of sensors in the everyday objects of our lives (Paravati and Gatteschi 2015).

\subsection{Types of Sensors:}

Sensors can be classified based on power or energy supply requirement of the sensors as passive and active sensor.

\subsubsection{Passive sensor}

Passive sensors require an external power source to operate, which is called an excitation signal. The signal is modulated by the sensor to produce an output signal. For example, a thermistor does not generate any electrical signal, but by passing an electric current through it, its resistance can be measured by detecting variations in the current and/or voltage across the thermistor (Fraden, 2016).

\subsubsection{Active sensor}

Active sensors generate electric signals in response to an external stimulus without the need of an additional energy source. Examples of active sensors are photodiode, piezoelectric sensor and a thermocouple (Kalantar-zadeh, 2013).

Another way of classifying sensors is with regards to the physical property they measure. The most common categories include Mechanical, Thermal, Electrical, Magnetic, Radiation, Chemical and Bio-chemical. Sensors can alternatively be classified according to the following criteria primary input quantity (for measurement), transduction principles (using physical and chemical effects), material and technology, property and application

\subsection{Functionality of Sensors}

According to Spencer et al (2014), for sensor to function and communicate, it has to be integrated with other component. A traditional integrated sensor can be divided into three parts as shown in figure 1

(i) The sensing element (e.g photo diode, light dependent resistor, piezo-electric material)

(ii) Signal conditioning and processing element (e.g for amplifications, linearization, compensation, and filtering)

(iii) A sensor interface (e.g. the wires, plugs and sockets to communicate with other electronic components)

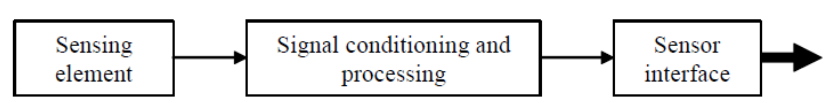

Figure 1: Traditional Integrated Sensors

Source: (Spencer, Manuel, \& Narito, 2014)

Table 1: Examples of Sensors with Their Measured

Property and Application type

\begin{tabular}{|c|c|c|}
\hline Sensor & $\begin{array}{l}\text { Measured } \\
\text { Property }\end{array}$ & $\begin{array}{l}\text { Application } \\
\text { Type }\end{array}$ \\
\hline Accelerometer & Acceleration & $\begin{array}{l}\text { Activity sensing, } \\
\text { Context sensing, } \\
\text { Environment } \\
\text { sensing, } \\
\text { Physiological } \\
\text { state sensing }\end{array}$ \\
\hline Air pollutants sensors & $\begin{array}{l}\text { Amount of toxic } \\
\text { particles in the } \\
\text { atmosphere }\end{array}$ & $\begin{array}{l}\text { Context sensing, } \\
\text { Environment } \\
\text { sensing }\end{array}$ \\
\hline Barometer & Pressure & $\begin{array}{l}\text { Activity sensing, } \\
\text { Context sensing, } \\
\text { Physiological } \\
\text { state sensing }\end{array}$ \\
\hline Bluetooth & Radio signals & $\begin{array}{l}\text { Activity sensing, } \\
\text { Context sensing }\end{array}$ \\
\hline Camera & Visual light & $\begin{array}{l}\text { Activity sensing, } \\
\text { Context sensing, }\end{array}$ \\
\hline
\end{tabular}




\begin{tabular}{|c|c|c|}
\hline & & $\begin{array}{l}\text { Environment } \\
\text { sensing, } \\
\text { Physiological } \\
\text { state sensing }\end{array}$ \\
\hline Compass & $\begin{array}{l}\text { Earth magnetic } \\
\text { field }\end{array}$ & Activity sensing \\
\hline $\begin{array}{l}\text { Electro cardiogram } \\
\text { (ECG or EKG) }\end{array}$ & Heartbeat & Activity sensing \\
\hline $\begin{array}{l}\text { Electromyography } \\
\text { sensor }\end{array}$ & $\begin{array}{l}\text { Electrical } \\
\text { activity } \\
\text { produced by } \\
\text { skeletal muscles }\end{array}$ & Activity sensing \\
\hline Gyroscope & $\begin{array}{l}\text { Measures } \\
\text { orientation }\end{array}$ & $\begin{array}{l}\text { Activity sensing, } \\
\text { Context sensing, } \\
\text { Physiological } \\
\text { state sensing }\end{array}$ \\
\hline Humistor & $\begin{array}{l}\text { Detects } \\
\text { humidity }\end{array}$ & Activity sensing \\
\hline Infra-red camera & $\begin{array}{l}\text { Infra-red } \\
\text { frequency of } \\
\text { light }\end{array}$ & $\begin{array}{l}\text { Activity sensing, } \\
\text { Context sensing }\end{array}$ \\
\hline $\begin{array}{l}\text { Radio frequency } \\
\text { identification } \\
\text { receiver }\end{array}$ & Radio frequency & Context sensing \\
\hline Sonar & $\begin{array}{l}\text { Detect objects } \\
\text { through sound } \\
\text { waves }\end{array}$ & Activity sensing \\
\hline Software Sensors & $\begin{array}{l}\text { Detect user's } \\
\text { actions }\end{array}$ & Context sensing \\
\hline $\mathrm{WiFi}$ & Radio frequency & Context sensing \\
\hline
\end{tabular}

\subsection{FACTORS TO CONSIDER IN} CHOOSING A SENSOR

Factors to consider in making the right choice of sensors for your specific circumstances include the following:

Geometry - The sensor needs to be the appropriate shape and size to fit your needs.

Measurement range - The sensor needs to be built to handle the appropriate range of measurement, whether it's temperature, pressure, flow, humidity, or another measurement.
Connectivity - The sensor needs to be able to easily connect to data loggers and other data acquisition and control equipment.

Deployment Environment - When implementing a wireless sensor system, understand the deployment environment to determine the topology of your network, the geographical location of measurement nodes,

Software and Data Access - A final factor to consider when implementing a wireless sensor is the software to be used to collect, analyse and present measurement data to local or remote clients.

\subsection{Wireless Sensors Security Requirements}

Many sensor networks are have mission-critical tasks to accomplish, hence there is a need to consider the security requirements at design stages. According to Bokare et al (2012) security requirements for any sensor architecture should include the following:

- Authentication: Since sensors use a shared wireless communication medium, authentication is necessary to enable sensor nodes to detect maliciously injected or spoofed packets. Authentication enables a node to verify the origin of a packet (source authentication) and ensure data integrity, that is, ensure that data is unchanged (data authentication).

- Secrecy: Ensuring the secrecy of sensed data is important for protecting data from eavesdroppers. Standard encryption functions can be used to achieve secrecy.

- Countermeasures: Standard cryptographic techniques can protect the secrecy and authenticity of communication links from outsider attacks such as eavesdropping, packet replay attacks, and modification or spoofing of packets.

- Key Establishment: for two sensor nodes to set up a secret and authenticated link, they need to establish a shared secret key.

- Broadcast Authentication: in broadcast source authentication, possible approach is to use a digital signature, where the source signs each message with a private key and all the receivers verify the message using the public key.

\subsection{CHALLENGES WITH WIRELESS SENSORS}

Despite the advancements and the breakthrough experienced with wireless sensors, major issues still plague the design and performance of wireless sensors. Below are some of the challenges:

2.5.1 Energy: Sensors require power for various operations. Energy is consumed in data collection, data processing, and data communication; also, continuous listening to the medium for faithful operation demands a large amount of energy by node components (CPU, radio, etc.) even if they are idle. 
Batteries providing power need to be changed or recharged after they have been consumed. Sometimes it becomes difficult to recharge or change the batteries because of demographic conditions.

2.5.2 Self-Management: Wireless sensors once deployed should be able to work without any human intervention. It should be able to manage the network configuration, adaptation, maintenance, and repair by itself. This is rarely achievable as physical management of the nodes are required to ensure they are properly positioned and capturing the correct data

2.5.3 Operating System: Operating System for Wireless Sensors should be less complex than the general operating systems and should have an easy programming paradigm.

2.5.4 Quality of Service (QoS): Wireless sensors are being used in various real time and critical applications, so it is mandatory for the wireless sensor to provide good QoS. Though, it is difficult because the network topology may change constantly and the available state information for routing is inherently imprecise.

2.5.5 Security: Confidentiality is required in sensor networks to protect information traveling between the sensor nodes of the network or between the sensors and the base station; otherwise it may result in eavesdropping on the communication.

2.5.6 Data Collection and Transmission: Data gathering is the main objective of sensor nodes. The sensors periodically sense the data from the surrounding environment, process it and transmit it to the base station or sink. Sometimes the sample of data collected is redundant and there is no need of transmitting such samples to the sink node as it will only consume energy. So care must be taken during data collection and transmission.

\section{HUMAN COMPUTER INTERACTION (HCI)}

Human computer Interaction (HCI) is defined as a discipline concerned with the design, evaluation and implementation of interactive computing systems for human use and with the study of major phenomena surrounding them (Hewett et al, 1996).

HCI has long been a focal area for innovative, multidisciplinary computing research and development. (Carroll, 2002). HCI consists of three parts: the user, the computer itself, and the ways they work together (Preece, 1994):

The goals of HCI are to produce usable and safe systems, as well as functional systems. These goals can be summarized as 'to develop or improve the safety, utility, effectiveness, efficiency and usability of systems that include computers'. Human Computer Interaction must also improve the interactions between users and computers by making computers more operational and receptive to the user's wants. Finally, HCI must develops or improves certain goals in designing devices.

\subsection{STRUCTURE AND SCOPE OF HCI}

HCI, as the name suggests, comprises three major parts within the framework: the user, the computer, and the interaction, indicates the ways they work together to achieve goals. Figure 2 below shows three main components of human computer interaction.

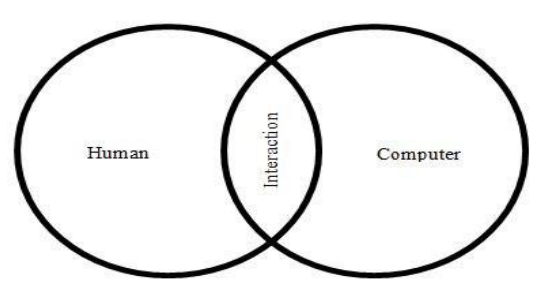

Figure 2: HCI Components

Human-Computer Interaction studies how people design, implement and use computer interfaces (Hollan, 2003). HCI has become an umbrella term for a number of disciplines including theories of education, psychology, collaboration as well as efficiency and ergonomics (Hinze-Hoare, 2007).

\subsection{HCI AND USER ACTIVITY INTERACTION LEVELS}

The field of HCI continues undergoing fast changes with the development of new multi-sensory user interface and metaphors. Smart devices, information processing engine, and large interactive displays have become more permeate (Gouin \& Lavigne, 2010). It has been widely accepted that the user activity has three interaction levels: physical, cognitive, and affective.

\subsubsection{Physical}

Te'eni et al (2007) in their book pointed out that the physical aspect combines the study of human body mechanics and physical limitations with industrial psychology to reach a fit between human and computer and accordingly improving the performance and the user's well-being.

.Moreover, the existing physical technologies for HCI generally can be organized by input and output devices. Input devices are the most obvious interactive technologies. These devices are generally organized around the human senses including vision, audition, and touch.

3.2.1.1 Vision. Vision is an important aspect in computer usage. It is the human process of seeing and comprehending objectives (Te'eni et al., 2007). For example, the size of the characters, the color of the foreground and background on the screen, and the angle and brightness of screen are all aspects of vision that may affect the interaction between users and computers.

3.2.1.2 Audition. The sense of hearing also plays an important role in human performance. Speech recognition is generally considered as the ability of computer to recognize 
human speech, usually with an audition device. Those difficult-to-build devices require advanced technologies to narrow the gap between human and computer. Alarms, beeps, and turn-by-turn navigation commands of a GPS device are common examples (Karray et al, 2008).

3.2.1.3 Touch. The sense of touch helps human beings understand the world at large. Touch is extremely important for the visually impaired. The keyboard and other direct manipulation devices such as the mouse have some relation to touch (Te'eni et al., 2007).

\subsubsection{Cognitive}

The cognitive aspect of interaction deals with ways of human understanding and interacting with computer systems. Cognitive engineering is a discipline that applies the combined knowledge of cognitive psychology and information technology to the design of artifacts. It aims to reduce complexity of interaction (Te'eni et al., 2007).

\subsubsection{Affective}

Affective refers to psychological processes and states including feelings, emotions, attitudes, satisfaction, and impressions. The affective aspect of interaction attempts to make the interaction with a cheerful experience and to affect the user to continue using the machine by changing their attitudes and emotions.

\subsection{HCI Modality}

An interface mainly relies on number and diversity of its inputs and outputs which are communication channels that enable users to interact with computer via this interface. Each of the different independent single channels is called a modality [Jaimes et al, 2007]. A system that is based on only one modality is called unimodal. Based on the nature of different modalities, they can be divided into three categories:

- Visual based: This include facial expression analysis, body movement tracking, gesture recognition, gaze detection (eye movement tracking).

- Audio based: This include speech recognition, speaker recognition, auditory emotion analysis, human-made noise detections (gasp, sigh, laugh, cry, etc) and musical interaction.

- Sensor based: The commonality of these areas is that at least one physical sensor is used between user and machine to provide the interaction. These include: Pen-based interaction, mouse \& keyboard, Joysticks, Motion tracking sensors and digitizers, haptic sensors, pressure sensors, taste/smell sensors

\subsection{Multimodal HCI Systems}

Multimodal is combination of multiple modalities. The modalities mostly refer to the ways that the system responds to the inputs or the communication channels. Multimodal interface acts as a facilitator of human-computer interaction through two or more nodes of input. It is expected that multimodal HCI system is combination of single modalities that interact correlatively.

An interesting aspect of multimodality is the collaboration of different modalities to assist the recognitions. For example, lip movement tracking (visual-based) can help speech recognition methods (audio-based) and speech recognition methods (audio-based) can assist command acquisition in gesture recognition (visual-based). Examples of applications of multimodal systems are: Smart Video Conferencing, Intelligent Homes/Offices, Driver Monitoring, Intelligent Games and applications for helping people with disabilities.

\section{REVIEW OF RELATED WORKS}

Bhagwani, Sengar \& Talwaniper (2012) developed a model Brain Computer Interface (BCI) tending toward the development of a device that can be controlled by thoughts, such as ability to change channels with mind or thought by capturing brain signals and translating them into commands that allow humans to control (just by thinking) devices such as computers, robots, rehabilitation technology and virtual reality environments. BCIs are often aimed at assisting, augmenting, or repairing human cognitive or sensory-motor functions.

Venkata and Neelima (2014) developed a tilt sensor computer controlled mouse which consist of an economical head operated computer mouse for people with disabilities as shown in figure 3 . It focuses on the invention of a head operated computer mouse that employs one tilt sensor placed in the headset to determine head position and to function as simple head-operated computer mouse. The system uses accelerometer based tilt sensor to detect the user's head tilt in order to direct the mouse movement on the computer screen. Clicking of mouse is activated by the user's eye brow movement through a sensor. The keyboard function is designed to allow the user to scroll letters with head tilt and with eye brow movement as the selection mechanism. Voice recognition section is also present in the head section to identify the small letters which are pronounced by the paralyzed user.

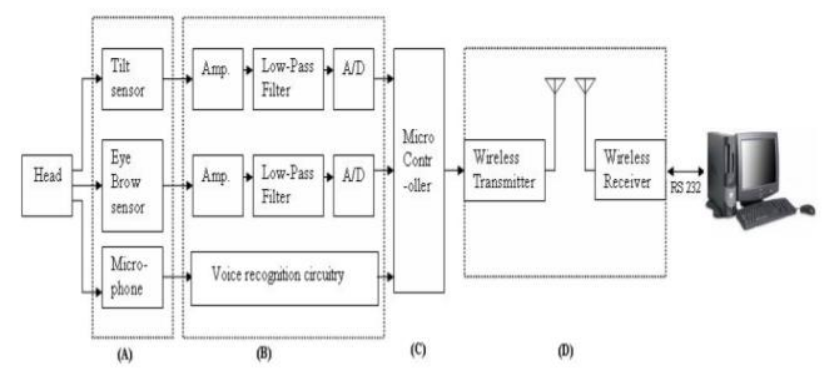

Figure.3. Block diagram representation of the tilt sensorscontrolled computer mouse interface. Source: Venkata and Neelima 2014 
Rajangam, Tseng, Yin, Lehew, Schwartz, Lebedev, and Nicolelis (2016) showed that rhesus monkeys can learn to navigate a robotic wheelchair, using their cortical activity as the main control signal. Two monkeys were chronically implanted with multichannel microelectrode arrays that allowed wireless recordings from ensembles of premotor and sensorimotor cortical neurons. Initially, while monkeys remained seated in the robotic wheelchair, passive navigation was employed to train a linear decoder to extract 2-Dimesionl wheelchair kinematics from cortical activity. Next, the monkeys were able to translate their cortical activity into the robotic wheelchair's translational and rotational velocities. Over time, monkeys improved their ability to navigate the wheelchair toward the location of a grape reward. These can be used to restore whole-body mobility to severely paralyzed patients in the future.

Chen, Xue, Mei \& Oetomo (2016) presented a state-of-art of wearable system for infant movement monitoring. The system showed how Neonatologist can give appropriate treatment and provide optimal nursing condition through the aid of wearable sensor. This is done by using sensor to monitor the body movement in infants as shown in figure 4

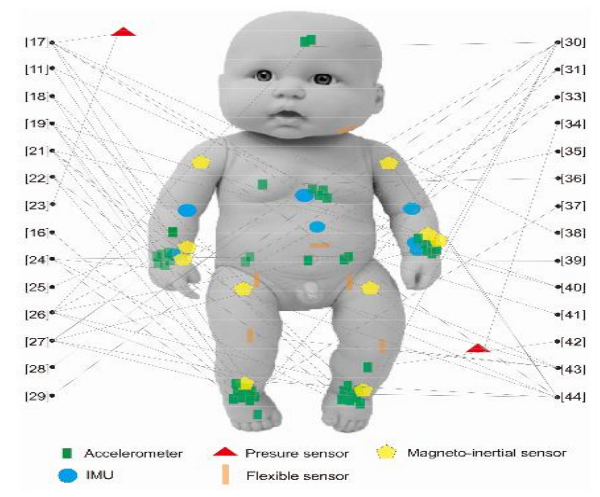

Figure 4: Infographic of sensor placement (Chen, Xue, Mei \& Oetomo 2016)

(Prange, Chikobava, Poller, Barz, \& Sonntag (2017) designed an interactive, real-time decision support for medical domain where physician and patient interact in virtual reality environment by using natural speech and hand gestures. The system involve the use of mobile tablet with stylus by radiologist to transcribe data in real-time using automated handwritten recognition, parsed, and representation based on medical ontologies. It creates a fully digital version of the radiology findings (mammography). The system is with a highly flexible architecture which can be connected to the existing hospital database systems (e.g, a picture archiving and communication system) and connects interaction devices such as Virtual Reality glasses and head-mounted displays.

Sandeep Kumar Singh et al (2018) investigated an animalhuman cohabitation problem with the help of machine learning and fibre-wireless access networks integrating cloud and edge computing, and then proposed an early warning system which detects wild animals nearby road/rail with the help of wireless sensors and alerts passing vehicles of possible animal crossing.

\section{HCI AND HUMAN ABILITIES}

The growing importance of human computer interaction (HCI) is a result of the widespread deployment of computers connecting with humans. The enhancement of human senses electronically is possible when pervasive computers interact unnoticeably with humans in ubiquitous computing.

Ubiquitous Computing regarded as "The Computer for The 21 st Century" even envisioned future computers to be invisibly living with humans, thereby widening the range of user types. With the popularity of the internet in recent decades, the concept of internet of things (IoT) further boosts the applications of ubiquitous computing to connect almost everything electronic to the Internet, with the ultimate result for an unprecedented demand for better HCI technology to cope with the needs for the huge number of non-technical users interacting with billions of network-connected computers.

\subsection{Natural user interface (NUI) Versus Disappearing User Interface (DUI)}

Human computer interaction is a multidisciplinary technology that heavily focuses on human-centric user interface design. An emerging trend in HCI is the design of Natural user interface (NUI) where humans can interact naturally with computers instead of using conventional methods such as command line interface (CLI) or graphical user interface (GUI), NUIs have been criticised as being "artificial naturallity" and as not representing natural human behaviours especially gesture based interactions (Malizia and Bellucci 2012) Hence, the introduction of the Disappearing User Interface (DUI) as a logical provision of natural HCI. DUI are tangible and intangible user interfaces that exist and work well but are not noticeable by humans, and are classified into three main categories:

- Human Body as DUIs

- Edible and Implantable DUIs

- Wearable DUIs

DUI is a natural HCI focusing on interaction with the contents instead of the interfaces (Lim 2012). Humans are content receivers through the sensory systems, and at the same time humans are content providers mainly through muscular movements and nervous systems. DUIs enable the conveyance of contents between humans and machines in a natural way through tangible or intangible artefacts; touch or touchless interface; outside or inside human bodies; and most importantly not noticeable by the target persons so the interaction is done based on intuition. DUI is multi-modal in nature by sensing human behaviours through different disappearing user interfaces in a natural way and creating the contents for exchange.

DUIs may interact with human inputs mainly through the five basic senses: vision, hearing, touch, smell and taste.

Humans do have more than five senses and they all fall into the same "stimulus-sensation-perception" process with the human brain as the final decision maker after interpreting the outside world from the stimuli. 
Figure 5 shows a typical illustration of a human sensory system and it can be seen that human perception based on the human brain is the result of decoding the sensation signals which are activated by the stimuli coming from the outside world through the basic sensory organs, while human outputs are the responses to this perception.

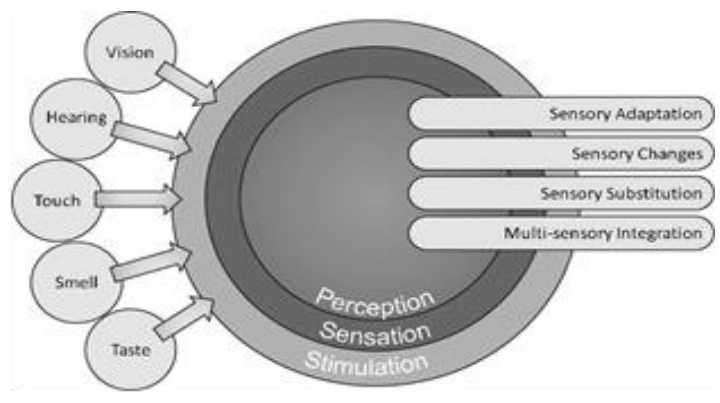

Figure 5: The Human Senses

Hearing is another popular human input interface, while computer generated sound in the audio frequency range is delivered through a medium to the human auditory system. Sonic interface is a good DUI for naturally interfacing with humans in open space, and the critical requirement is the delivering of high quality sound signals which can normally be achieved through frequency response equalisation. The sense of touch also enables a private communication with computers through the human skin.

Human smell, which is a chemical sense is another human input interface. Presentation of smell stimuli can be achieved through diffusion to open air but the vaporisation of odour molecules is relatively slow compared to the propagation of mechanical waves in sound and electromagnetic waves in vision, thus a continuous emission of odour vapour is normally applied.

Despite that the nature of stimulus to each type of sense organ is different, the perception can be the same. Sensory Substitution is an emerging research demonstrating that Brain Plasticity enables human brain to choose a particular type of sensor or combination of sensors to arrive at the same perception after a training period (Bermejo and Arias 2015). DUI should therefore be more effective to interface with human inputs through delivering content instead of raw data, and the more we know about the human brain, the more we will realise how to interface with humans.

DUIs also capture human outputs based on monitoring human body activities: body parts movements, body sound generation, body temperature variations, body odour emissions, and the dynamic physiological parameters. The disappearing nature of DUI avoids step-by-step manual driven human interaction, and its focus is set on interfacing with the contents which are the actions generally derived from human perceptions (Lim 2012).

\section{IMPROVEMENT OF HUMAN ABILITY WITH HCI}

For many years, humans have sent commands to "machines" primarily via the keyboard-mouse paradigm also known as WIMP (windows, icons, menus, point-and-click devices). Here, the term machine is used in a very broad sense: in addition to the point-and-click devices that are usually associated with computers, a keyboard of sorts is used to dial numbers on a telephone, to interact with a TV, to select a wide range of functions on a car dashboard, and many other activities that employ key-based interaction modalities. In most cases, the machine's output to the user is then based on a display device such as a monitor.

As foreseen by Andy van Dam, in his vision paper published in IEEE Computer Graphics \& Applications' first issue of the new century: "Post-WIMP interfaces will not only take advantage of more of our senses but also be increasingly based on the way we naturally interact with our environment and with other humans."

Several affordable sensors have begun to shake up the way people interact with devices. Touch and multi-touch screens have driven the change from cellular phones to smartphones, and gestures are now the main interaction modality to activate functions on personal devices. At the same time, speech recognition technologies and CPUs' increased computational power now let users efficiently provide inputs when they can't perform gestures.

Personal devices are the most evident example of how new forms of HCI can reduce the gap between humans' mental models and technology. One market that has led this deep innovation in $\mathrm{HCI}$ is entertainment. With users asking game and device makers for new ways to control characters, game console developers proposed controllers to release players from the constraints of using a keyboard and a mouse. The new interface becomes a means for providing tactile feedback as well as acting as a sort of tangible interface (the controller becomes a steering wheel, a gun, or a tennis racket).

Sensors such as the Microsoft Kinect are a further step toward the implementation of fully natural interfaces in which the human body becomes the controller. The device lets users provide commands to the machine via gestures and body poses as embedded hardware performs real-time processing of raw data from a depth camera, thus obtaining a schematic of a human skeleton comprising a set of bones and joints. Recognizing the position and orientation of bones lets the hardware identify poses and gestures, which can be mapped to commands for the machine.

Researchers have also proposed sensors that can track a user's hands. For instance, the Leap Motion can interactively track both hands of a user by identifying the positions of finger tips and the palm centre. Some car makers are already proposing a hand-tracking based alternative interaction modality in lieu of traditional touch screens devoted to managing infotainment functions. Similarly, some smart Televisions let users control their choices with a set of gestures, thus replacing the traditional remote control.

Found only in science fiction movies just a few years ago, the above-mentioned scenarios are now the present reality of HCI. On the other hand, new and more intriguing scenarios appear to be imminent, as brain interfaces seem poised to invert the relationship between humans and machines, for instance. This new interaction paradigm's success will rely on future technological advances, which aim to transform interface devices into wearable and embeddable objects. 
Interfaces based on augmented reality (AR) technologies are clear examples of this transformation. Many applications for tourism, entertainment, maintenance, shopping, and social networks are already available for personal devices, but new wearable sensors might soon change our habits.

Eye-tracking is also replacing tasks usually performed by the mouse. Eye-tracking can help in picking up the cues that are subconsciously given by the other person and hence, in enhancing the overall interaction Eyes can reveal a lot about a person's intentions, thoughts and actions, as they are good indicators of what we're interested in. There's a lot of potential in eye-tracking in other arenas as well.

Wearables, the Internet of Things and Smart Materials are realms that are opening ways for even more eccentric and unimaginable things, into a future with great improvement on human abilities with $\mathrm{HCI}$

\section{CONCLUSION}

This paper reviewed the applications of sensors in HCI, how integrating sensors with human ability enhances the possibilities of HCI and what can be done. HCI can be implemented virtually in all spheres of life and offer opportunities to make life comfortable and enjoyable. New forms of HCI with the use of wireless sensors will change our lives significantly and improve the quality of life of people who cannot take advantage of current interfaces due to physical disabilities. The opportunities for HCI are tremendous with progress made toward more user-friendly and natural interfaces for human-machine interaction. Finally, further research on the integration of sensors with HCI will help to solve many problems in future.

\section{FUTURE OF HCI}

The future of HCI is anchored on multimodality, intelligent, adaptive interfaces and active interfaces. Multi-modal interfaces attempt to address the problems associated with purely auditory and purely visual interfaces by providing a more immersive environment for human-computer interaction.

The interfaces of the future should be Non-Windows, Icons, Menus, Pointer (Non-WIMP) and more of natural features than artificial. HCI must give special attention for language understanding, mobile and handheld Interaction for rich user experience and high level of functionality.

HCI must think beyond audiovisual Interfaces by providing new tools for interactive experience design and also help to uncover sensory stimulation and emotional responses that can be used in the future for better human computer interaction. There is still a lot of challenge in understanding people's multisensory experiences in HCI because the senses we call upon when interacting with technology are restricted. Another future goal is to understand the ways in which our senses process information and how they relate to one another to create richer experiences for human-technology interactions.

\section{ACKNOWLEDGMENTS}

Our thanks to the current and previous students of Human Computer Interaction and Sensors Research group of the Computer Science Department of the School of Computing and Engineering Sciences, Babcock University, Ilishan-Remo, Nigeria who have contributed towards the development of this paper.

\section{REFERENCES}

1) Bermejo F, Di Paolo EA, Hüg MX and. Arias C, Sensorimotor strategies for recognizing geometrical. shapes: a comparative study with. different sensory substitution devices : Frontiers in Psychology 6, 10.3389/fpsyg.2015.00679

2) Bhagwani, A, Sengar, C and Talwaniper, J (2012). Human Computer Interaction. International Journal of Advancements in Research \& Technology Vol 1 Issue 3 pp 72-76

3) Bokare, $M$ and Ralegaonkar, A (2012). Wireless Sensor Network: A Promising Approach for Distributed Sensing Tasks; Excel Journal of Engineering Technology and Management Science, Vol. I No.1; ISSN 2249-9032

4) Carroll., J (2002)."Human-Computer Interaction in the New Millenium," ISBN-13: 978-0-20170447-1

5) Chen, H; Xue, M;Mei, Z; Bambang Oetomo, S; Chen, W (/2016). A review of wearable sensor systems for monitoring body movements of neonates. Sensors 2016, 16,2134; doi : $10.3390 / \mathrm{s} / 16122134$

www.mdpi.com/journal/sensors

6) Dargie, W, Poellabauer,C (2010). Fundamentals of wireless sensor networks: theory and practice. John Wiley \& Sons.

7) Fraden J, 2016: Handbook of Modern Sensors, Available https://doi.org/10.1007/978/3/319/19303/8/1

8) Fritzsche, B. (2015). Human Computer Interaction in the Internet of Things Era. Munich: University of Munich Department of Computer Science Media Informatics Group.

9) Gouin, D. \& Lavigne, V. (2010). Trends in humancomputer interaction to support future Intelligence analysis capabilities. Paper presented at the Sixteenth International Command and Control Research and Technology Symposium, Quebec City, Canada. 
10) Hewett, Baecker, Card, Carey, Gasen, Mantei, Perlman, Strong and Verplank, "ACM SIGCHI Curricula for Human-Computer Interaction," 1996. http://old.sigchi.org/cdg/cdg2.html

11) Hinze-Hoare, V. ( 2007). "Review and Analysis of Human Computer Interaction (HCI) Principles", Southampton University, pp.2

12) Hollan, J. D. (2003). Human-Computer Interaction",The MIT Encyclopedia of the Cognitive Sciences, Robert Wilson and Frank Keil (Eds), MIT Press, San Diego, pp.1

13) Hua, K, Yea, J, Fana, E, Shena, S, Huanga, L, Pi, J (2017). A novel object tracking algorithm by fusing color and depth information based on single valued neutrosophic cross-entropy. Journal of Intelligent \& Fuzzy Systems (32), 1775-1786.

14) Jaimes, A and Sebe, N 2007 "Multimodal human computer interaction: a survey", Computer Vision and Image Understanding, 108(1-2), pp 116-134.

15) Kalantar-zadeh (2013). Sensors: An Introductory Course 2013 Edition, Kindle Edition. by Kourosh Kalantar-zadeh ebook . Available at https://www.amazon.com/Sensors-

Introductory...Kalantar-zadeh.../B00C6BZROU

16) Karray, F., Alemzadeh, M., Saleh, J. A., \& Arab, M. N. (2008). Human-computer interaction: overview on state of the art. International Journal on Smart Sensing and Intelligent Systems, 1(1), Pp. 137-159.

17) Lim YK (2012) Disappearing interfaces. Interactions 19(5):36-39. doi:10.1145/2334184.2334194

18) Malizia and Bellucci 2012; The Artificiality of Natural User Interfaces: Communications of the ACM 55(3):36-38 - March 2012 https://dl.acm.org/citation.cfm? id $=2093563$

19) Paravati, G. Gatteschi, V, (2015). Human-Computer Interaction in Smart Environments. Sensors, 15, 19487-19494.

20) Prange, A, Chikobava, M, Poller, P, Barz, M, Sonntag, D (2017). A Multimodal Dialogue System for Medical Decision Support in Virtual Reality. Proceedings of the SIGNAL, Conference, pages 2326. Association for Computational Linguistics.

21) Preece, J. (1994), "Human Computer Interaction", Addison Wesley pp.6, 26

22) Rajangam, Tseng, Yin, Lehew, Schwartz, Lebedev, and Nicolelis (2016): Wireless Cortical BrainMachine Interface for Whole-Body Navigation in Primates. National Center for Biotechnology Information, U.S. National Library of Medicine.
Available

at

https://www.ncbi.nlm.nih.gov/pubmed/26938468

23) Sandeep Kumar Singh et al (2018): Improving Animal-Human Cohabitation with Machine Learning. Available at: https://www.mdpi.com/2224-2708/7/3/35/pdf

24) Spencer F., Manuel E., \& Narito K., (2014) Smart Sensing Technology: Opportunities and Challenges. Structural Control and Health Monitoring 11(4):349 368 Available https://www.researchgate.net/.../227533576_Smart_ Sensing_Technology_Opportunities

25) Sunny, George and Kizhakkethottam, (2015): Applications and Challenges of Human Activity Recognition Using Sensors In A Smart Environment: International Journal for Innovative Research in Science \& Technology. Volume 2 Issue $-4$

26) Te'eni, D., Carey, J., \& Zhang, P., (2007). Human computer interaction developing effective organizational information systems. Hoboken John Wiley \& Sons,

27) Varkey, J., Pompili, D., \& Walls, T. (2012). Human motion recognition using a wireless sensor-based wearable system. Personal \& Ubiquitous Computing, 16(7), 897-910.

28) Venkata, M and Neelima, B. 2014:A Portable Wireless Head Movement Controlled HumanComputer Interface for People with Disabilities International Journal of Advanced Research in Electrical, Electronics and Instrumentation Engineering (An ISO 3297: 2007 Certified Organization) Vol. 3, Issue 7, July 2014 